\title{
Differentiation of Pseudomonads by Amplification of Metabolic Profiles
}

\author{
EDWARD J. HSU,* JAMES H. GODSEY, EDWARD K. CHANG, and SANDRA L. LANDUYT \\ Department of Biology, University of Missouri-Kansas City, Kansas City, Missouri 64110
}

Cultures of four species of Pseudomonas grown in a complex medium were shifted down to a minimal medium for assaying aminopeptidases and metabolic end products. Cultural conditions were further modified to include both agitation and stationary conditions. Growth rates were determined for each condition to obtain maximal cell yield. Cells were then harvested for intracellular aminopeptidase assay by fluorometric analysis of enzyme activity, and a gas-liquid chromatography analysis was made for extracellular metabolic end products. A high reproducibility was obtained from the aminopeptidase profiles of strains of Pseudomonas fluorescens, $P$. putida, $P$. stutzeri, and $P$. pickettii, including a control consisting of a blind test of previous isolates. The shift down to the minimal medium increased enzyme activity in each case and induced characteristic new peaks in most instances. Gas-liquid chromatography analysis was concentrated on short-chain volatile fatty acids no longer than seven carbons. Butyl ester derivatives were prepared to enhance separation and increase percent recovery of these volatile components. The resulting change in profiles under different physiological conditions indicates a new method for the possible identification of Pseudomonas species.

Automation in the identification of bacterial isolates has recently received a great deal of interest. Because of this, instrumental analysis of biological samples has become a standard procedure in many laboratories today. Two procedures requiring such instrumentation are the aminopeptidase assay and gas-liquid chromatography (GLC).

The aminopeptidase (arylamidase) procedure is based on the enzymatic liberation of fluorescent $\beta$-naphthylamine from a nonfluorescent $L$ amino acid- $\beta$-naphthylamide (AA-NA) (26). More recently the aminopeptidases of Saccharomyces cerevisiae have been shown to be largely overlapping in specificities, but they can be distinguished from one another by different peptide substrates (19). Westley et al. (27) examined 21 species of Bacillus and two substrains of Escherichia coli K-12. These workers found each organism to have a distinct order of attack on AA-NA substrates, beginning with their preferred substrate and ending with substrates used only in trace amounts. Also examined was the possibility of repressing the synthesis of specific aminopeptidases by incorporating certain amino acids into the minimal growth medium. Each microorganism assayed was grown on three different kinds of solid medium, and the growth was harvested with a wire loop. A modification of the growth in broth medium permitted the monitoring of relative numbers of cells in the culture and, thus, a more precise examination of several genera in the family Enterobacteriaceae (15).

Some questions as to the location of aminopeptidases have caused confusion among researchers as to whether one should sonicate bacterial cells to release intracellular aminopeptidases or incubate intact cells with the substrate to assay membrane-bound or extracellular aminopeptidases. Choules and Gray (1) found little aminopeptidase activity in the medium of $M y$ coplasma laidlawii cultures, although considerable aminopeptidase activity was assayed from both the membrane and the cytoplasm of these cells. Matheson et al. (11) described for $E$. coli $\mathrm{B}$ a ribosome-bound aminopeptidase which they proposed to be involved in the initiation step of protein synthesis.

Through experiments utilizing oligopeptides as inhibitors of aminopeptidase activity, two groups found the enzyme to be multisited, with the last binding site having a high affinity for bulky, hydrophobic residues $(6,25)$. Both groups also examined AA-NA derivatives as possible substrates and found that the bulky $\beta$-naphthylamine moiety enhanced binding to the enzyme.

In view of these facts, it was not surprising that AA-NA derivatives were chosen as the substrates for aminopeptidase assays. Along with identification of microorganisms, aminopepti- 
dase profiles could be used to select favorable growth media for pure bacterial strains.

Microorganisms were identified through GLC analysis of volatile and nonvolatile acids produced as metabolic end products during growth $(4,12)$ or of fatty acids extracted from whole cell hydrolysates. The extraction of monocarboxylic acids produced as metabolic by-products was the first procedure utilized $(5,9,24)$. Although very inefficient for short-chain volatile acids, this extraction worked for long-chain, nonvolatile acids. Analysis of butyl ester derivatives formed by the addition of borontrifluoride-1-butanol to saponified acids has been advocated (14). This procedure, unlike all of the previous procedures, allowed one to look at both shortchain volatile and nonvolatile monocarboxylic and dicarboxylic acids on the same chromatogram and, for the first time, allowed the resolution of formic acid by flame ionization detectors $(10,20)$. Doelle (3), Fugate et al. (5), and Mitruka (13) have reviewed many of the commonly used extraction procedures and have published them as flow charts, making it now possible to analyze many different types of bacterial metabolites by GLC. Just as important as the derivatization procedure was the stationary phase chosen for doing the separation of volatile components (3). The most promising stationary phase was the heat-stable Dexsil 300-GC (10). For this reason, GLC analysis performed in the present study utilized butyl ester derivatives separated on a Dexsil 300-GC stationary phase. Although GLC analysis offers high sensitivity and rapid detection of most cellular components and products, it also has the disadvantage that accurate identification is rarely possible without the concurrent use of additional techniques.

Cultures of Pseudomonas species such as $P$. aeruginosa, $P$. fluorescens, $P$. pseudomallei, $P$. putida, and $P$. stutzeri have been isolated from infections of the ear canal, middle ear, urinary tract, various types of wounds, and as contaminants of transfusion blood $(7,17)$. Although the pathogenicity of these pseudomonads was not clearly established, their presence among clinical isolates cannot be overlooked. The ability of gram-negative rods to transfer antibiotic resistance on extrachromosomal deoxyribonucleic acid, i.e., plasmids, makes this particular group of bacteria very significant because of their high frequency of isolation as hospital contaminants $(2,8,18)$. Pseudomonads are nutritionally versatile and use a variety of organic compounds as sole carbon sources. Recently, several plasmids that confer the ability to degrade octane, camphor, salicylate, xylene, or naphthalene and the ability to utilize amino acids were isolated from strains of P. putida (2) and P. aeruginosa (8), respectively.

Stanier et al. (23) examined 147 biochemical, nutritional, and morphological characters in their classic characterization of this genus. An additional 40 physiological characters were used by Sands et al. (22) in grouping the phytopathogenic pseudomonads. In an effort to simplify the matter, Pickett and Pederson $(16,17)$ chose 17 primary and secondary features for the identification of most clinical isolates known as nonfermentative rods. A large battery of tests would not be necessary if secondary features of identification could be eliminated through semiautomated instrumental analysis. The biochemical basis of aminopeptidase assays and GLC analysis, when combined, may supersede the morphological, physiological, and pathogenic characteristics.

\section{MATERIALS AND METHODS}

Organisms. All cultures were obtained from M. J. Pickett, Department of Bacteriology, University of California, Los Angeles, and were designated by the numbers under which they were received. Strains of four different species of Pseudomonas were used in this study as follows: $P$. fluorescens strains K129, $\mathrm{K} 175$, K170, and K466; P. putida strains K89, K331, $\mathrm{K} 355$, and $\mathrm{K} 360$; P. stutzeri strains K607, K814, and K369; and P. pickettii strains K214, K232, and K279. Stock cultures were maintained under mineral oil on nutrient agar slants at $4^{\circ} \mathrm{C}$. New stock cultures were prepared every 3 months.

Cultural techniques. Different physiological conditions were established by using both complex and minimal growth media. Nutrient broth (Difco Laboratories), the complex medium, consisted of $3 \mathrm{~g}$ of beef extract and $5 \mathrm{~g}$ of peptone extract per liter. The minimal medium contained sodium acetate as the sole carbon source. The minimal medium contained, per liter: $4.0 \mathrm{~g}$ of sodium acetate; $11.8 \mathrm{~g}$ of $\mathrm{KH}_{2} \mathrm{PO}_{4} ; 8.8 \mathrm{~g}$ of $\mathrm{Na}_{2} \mathrm{HPO}_{4} ; 0.02 \mathrm{~g}$ of $\mathrm{NaCl} ; 0.24 \mathrm{~g}$ of $\mathrm{MgSO}_{4} ; 0.0028$ $\mathrm{g}$ of $\mathrm{FeSO}_{4}$; and $4.0 \mathrm{~g}$ of $\left(\mathrm{NH}_{4}\right)_{2} \mathrm{SO}_{4}$. To prevent precipitation, $\mathrm{FeSO}_{4}$ was dissolved and added separately after all other components had been dissolved. The $\mathrm{pH}$ of the medium was then adjusted to 7.0 before autoclaving.

A loopful of the stock culture was transferred into $8 \mathrm{ml}$ of nutrient broth. The culture was incubated overnight $(16$ to $18 \mathrm{~h})$ at $30^{\circ} \mathrm{C}$ in a water bath shaker. An exponentially growing culture $(3 \mathrm{ml})$ was then transferred to $70 \mathrm{ml}$ of nutrient broth. Each culture was incubated under one of two conditions: in the water bath shaker at $30^{\circ} \mathrm{C}$ or in a stationary water bath at $30^{\circ} \mathrm{C}$. Growth of the culture was monitored by determing the optical density by means of a Hitachi Perkin-Elmer 139 spectrophotometer set at $600 \mathrm{~nm}$.

The minimal medium $(8 \mathrm{ml})$ was inoculated with $0.1 \mathrm{ml}$ of an exponentially growing nutrient broth culture and was incubated at $30^{\circ} \mathrm{C}$ overnight (16 to 18 h) in a water bath shaker. A stoppered flask containing $70 \mathrm{ml}$ of minimal medium was then inoculated with 3 
$\mathrm{ml}$ of the overnight culture and incubated at $30^{\circ} \mathrm{C}$ under one of the above-mentioned conditions. For aminopeptidase assays and GLC analysis, when the optical density reached 0.85 growth was terminated by placing the culture in an ice bath for $10 \mathrm{~min}$. The cells were harvested by centrifugation at $10,000 \times g$ for 10 min at $5^{\circ} \mathrm{C}$ and washed three times in cold $0.9 \%$ saline. The cells were resuspended, and the optical density was adjusted to 0.85 in cold $0.9 \%$ saline. The cell suspension was again placed in an ice bath and sonicated with a Fisher Ultrasonic model BP 25. Duration of sonication was 1 to $2 \mathrm{~min}$ followed by cooling for 3 min, with the cycle being repeated until disruption was at least $95 \%$ complete. The sonicated cell suspension was centrifuged at $5,000 \times g$ at $5^{\circ} \mathrm{C}$ for $10 \mathrm{~min}$, and the supernatant was used as the crude enzyme preparation.

Aminopeptidase assay. The 19 substrates were AA-NAs. These nonfluorescent compounds were hydrolyzed to the highly fluorescent end product $\beta$-naphthylamine. Fluorometric determinations of the end product were used to obtain maximum sensitivity (15). Samples (2 ml each) of the AA-NA substrates were mixed with $0.1 \mathrm{ml}$ of crude enzyme preparation. These reaction mixtures were incubated in a $30^{\circ} \mathrm{C}$ circulating water bath for $1 \mathrm{~h}$ followed by heating in a $56^{\circ} \mathrm{C}$ water bath for $10 \mathrm{~min}$ to stop the reactions. One unit of aminopeptidase was the amount of enzyme that released $1 \mu \mathrm{mol}$ of $\beta$-naphthylamine per min (at $\mathrm{pH} 8.0$ and $30^{\circ} \mathrm{C}$ ) from the AA-NA. The $2.10 \mathrm{ml}$ of reaction mixture contained $10^{-4} \mathrm{M}$ AA-NA, $10^{-1} \mathrm{M}$ tris(hydroxymethyl)aminomethane-acetate buffer, and $7.7 \times 10^{-3} \mathrm{M} \mathrm{NaCl}$. With these conditions, an increase of 0.63 relative fluorescence intensity in the reaction mixture was equivalent to 1 enzyme unit.

Substrates were prepared in 1-liter quantities and stored at $4^{\circ} \mathrm{C}$. Substrate glassware was covered with aluminum foil to prevent decay. To further enhance the reproducibility of this procedure, accessory equipment is now available to eliminate direct contact and exposure of AA-NA substrates during fluorescenceintensity readings. Such accessories include a peristaltic pump to draw substrate into and discharge it from a microcell for fluorometric readings and an automatic sample changer to allow direct readings of cuvettes without manual transfer. The major advantage of the fluorometric assay technique is the increased sensitivity. Substrate concentrations can be reduced 1,000 times to reduce the cost for clinical laboratories adopting such a technique $(15,26)$.

Preparation of butyl ester derivatives. Butyl ester derivatives of monocarboxylic acid standards were prepared by the method of Lambert and Moss (10). The monocarboxylic acids derivatized were formic $\left(C_{1}\right)$, acetic $\left(C_{2}\right)$, propionic $\left(C_{3}\right)$, butyric $\left(C_{4}\right)$, iso-butyric (i-C $\left.\mathrm{C}_{4}\right)$, valeric $\left(\mathrm{C}_{5}\right)$, caporic $\left(\mathrm{C}_{6}\right)$, heptanoic $\left(\mathrm{C}_{7}\right)$, and lactic (lac) acid (Aldrich Chemical Co., Milwaukee, Wis.).

In the preparation of the actual sample, $4 \mathrm{ml}$ of spent medium was removed from the culture when the optical density reached 0.85 and was centrifuged at $12,100 \times g$ for $10 \mathrm{~min}$ at $5^{\circ} \mathrm{C}$. The supernatant was then removed to a $25-\mathrm{ml}$ serum bottle where the $\mathrm{pH}$ was adjusted to ca. 12 with $10 \mathrm{M} \mathrm{NaOH}$. This converted the free fatty acids into their salts, thus pre- venting their loss during the subsequent freeze-drying. The serum bottles were stoppered, and the alkalinized supernatants were shell-frozen in an ethanol-dry ice bath. The frozen samples were then placed in a Revco ultra-low freezer at $-70^{\circ} \mathrm{C}$ for $30 \mathrm{~min}$ to ensure that each sample was thoroughly frozen, thus preventing "meltback" during freeze-drying. All samples were transported to the freeze dryer in a styrofoam vessel containing dry ice. A Labconco freeze dryer 12 was used to freeze-dry all samples for $4 \mathrm{~h}$ at $-50^{\circ} \mathrm{C}$. The dried salts of the monocarboxylic acids were then transferred to a clean Teflon-lined, screw-capped tube (13 by $100 \mathrm{~mm}$ ) and dissolved in $1 \mathrm{ml}$ of chloroform. The monocarboxylic acids were butylated by adding $0.2 \mathrm{ml}$ of $14 \%$ (wt/vol) borontrifluoride-butanol (Applied Science Laboratories, St. College, Pa.). The reaction mixtures were thoroughly mixed on a Genie Vortex mixer and then tightly capped and heated in a Haak E52 circulating water bath at $90^{\circ} \mathrm{C}$ for $1 \mathrm{~h}$. Upon cooling to room temperature, $0.1 \mathrm{ml}$ of trifluoroacetic anhydride was added to each tube. The solutions were gently mixed and were allowed to react for $1 \mathrm{~h}$ at room temperature. The trifluoroacetylation step in the butylation procedure not only removed free $n$-butanol from the reaction mixture but also formed trifluoroacetyl esters with hydroxy acids such as lactate. The samples were washed with $2 \mathrm{ml}$ of distilled water to remove excess trifluoroacetic anhydride. The inorganic layer was discarded, and the chloroform layer, containing the butyl ester derivatives, was concentrated to $0.1 \mathrm{ml}$. Each sample $(2 \mu \mathrm{l})$ was injected onto the column for GLC analysis.

Conditions of GLC analysis. A Varian Aerograph 2740 gas chromatograph equipped with flame ionization detectors was used for analyses. Peaks were recorded on a Varian A-25 strip-chart recorder.

Separation of butyl ester derivatives was accomplished on a coiled stainless steel column $(1 / 8$ in. by 6 $\mathrm{ft}$ [ca. $0.31 \mathrm{~cm}$ by $1.82 \mathrm{~m}$ ]) packed with Chromosorb w-Hp (80/100 mesh, acid washed, dimethylchlorosilane treated) coated with $10 \%$ Dexsil 300-GC (Analabs, North Haven, Conn.). Columns were packed under reduced pressure with constant vibration. The ends of the column were plugged with silanized glass wool. Upon completion of packing, the column was gently coiled into its proper configuration. Columns were conditioned at $300^{\circ} \mathrm{C}$ overnight $(16 \mathrm{~h})$ under a minimum flow of nitrogen gas. The detector end of the column was left unconnected during the conditioning period. On-column sample injection was done manually with a $10-\mu l$ syringe.

During analyses, the injector port temperature was maintained at $275^{\circ} \mathrm{C}$, and the detector temperature was maintained at $300^{\circ} \mathrm{C}$. The column bath was held at $40^{\circ} \mathrm{C}$ for $8 \mathrm{~min}$ after injection of the $2-\mu \mathrm{l}$ sample and then temperature programmed to $170^{\circ} \mathrm{C}$ at $8^{\circ} \mathrm{C} / \mathrm{min}$. The carrier gas was prepurified nitrogen with a flow rate of $30 \mathrm{ml} / \mathrm{min}$. The flow rates of hydrogen and compressed air were 30 and $300 \mathrm{ml} / \mathrm{min}$, respectively, as measured by soap film flow meter. The electrometer range was set at $10^{-11} \mathrm{~A} / \mathrm{mV}$ with an attenuation of 32 . The total time for completion of one analysis was approximately $25 \mathrm{~min}$ followed by a cooling-off period of $6 \mathrm{~min}$.

To achieve more resolution of the esterified carbox- 
ylic acids, a program was developed for an initial isothermal period of $40^{\circ} \mathrm{C}$ for $8 \mathrm{~min}$ and increases of $8^{\circ} \mathrm{C} / \mathrm{min}$ to $170^{\circ} \mathrm{C}$. The initial temperature of $40^{\circ} \mathrm{C}$ could be obtained in a 6 -min cooling-off period by raising the door to the column oven. This 8-min hold at $40^{\circ} \mathrm{C}$ allowed a greater degree of separation of the formic acid peak from the solvent front. Temperature programming at the rate of $8^{\circ} \mathrm{C} / \mathrm{min}$ provided a relatively close and even separation of all standard acid peaks. Another advantage of temperature programming was that each analysis could be done within 25 min. In an effort to shorten further the elution time, a program rate of $10^{\circ} \mathrm{C} / \mathrm{min}$ was tested using the butyl ester standards. This new rate successfully reduced the elution time for one analysis, but the distance between neighboring peaks was also reduced, thus increasing the chance of incorrectly identifying unknown peaks.

In preparing the chloroform layer, which contains the butyl ester derivatives for analysis, it was important to note that if the final volume of the sample was less than $0.1 \mathrm{ml}$, a significant loss of $\mathrm{C}_{1}$ to $\mathrm{C}_{4}$ acids would occur due to evaporation. If the sample was evaporated to dryness and then again dissolved in 0.1 $\mathrm{ml}$ of chloroform, all nine acids failed to appear on the chromatogram. Whether this phenomenon was due to evaporation or degradation of the butyl esters was not ascertained, but it is most likely due to evaporation, as demonstrated by volatility during GLC analysis.

\section{RESULTS}

Aminopeptidase profiles in complex medium. Figure 1 shows distinctive profiles of strains of four different Pseudomonas species. As a group, the strains exhibited high alanine and arginine aminopeptidase activity. Under strictly controlled conditions, the profiles proved to be reproducible.

When grown in nutrient broth, all three strains of $P$. putida (Fig. 2) were characterized by high alanine, lysine, phenylalanine, arginine, leucine, proline, methionine, and glycine aminopeptidase activities, suggesting an order of attack on the AA-NA substrates. The remaining substrates were hydrolyzed only in small amounts with no recognizable pattern. In general, a distinguishing characteristic of the $P$. putida strains would be their high phenylalanine aminopeptidase activity.

The three strains of $\boldsymbol{P}$. fluorescens examined were not as homogeneous as those of P. putida. These strains preferentially hydrolyzed the $\beta$ naphthylamides of alanine, arginine, proline, lysine, phenylalanine, leucine, glycine, and methionine (Fig. 3). Each strain differed somewhat in

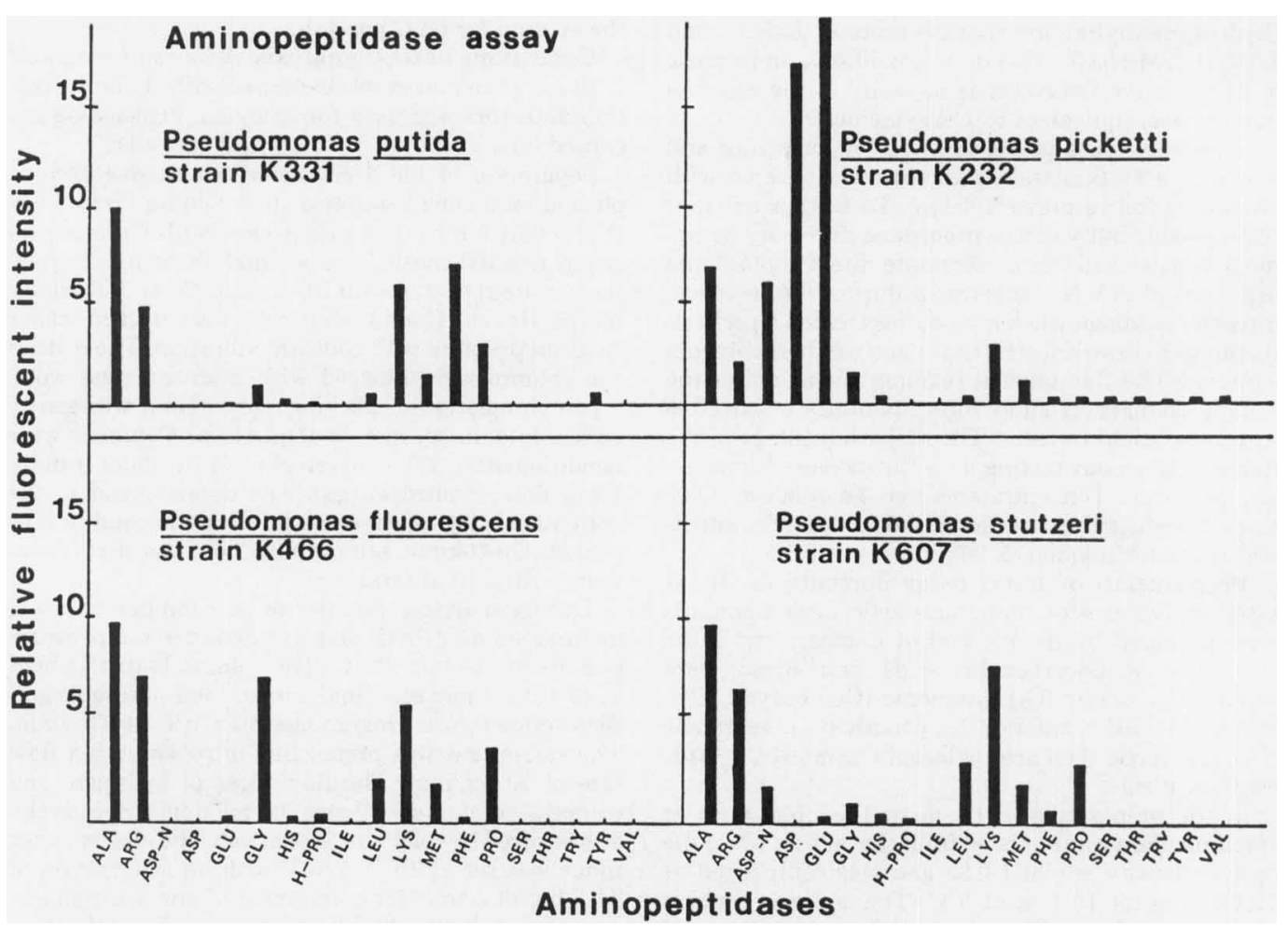

FIG. 1. Differentiation of Pseudomonas species by aminopeptidase activity. Cells were grown in complex medium and prepared as described in the text. 


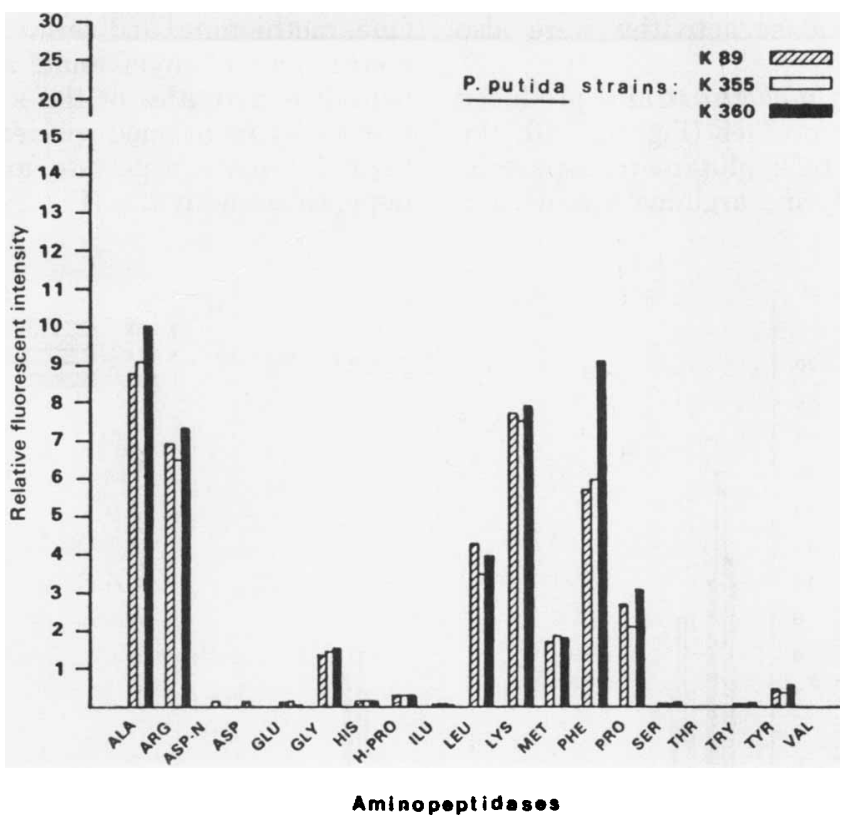

Fig. 2. Differentiation of three strains of $P$. putida by aminopeptidase activity. Cells were grown in complex medium and prepared as described in the text.

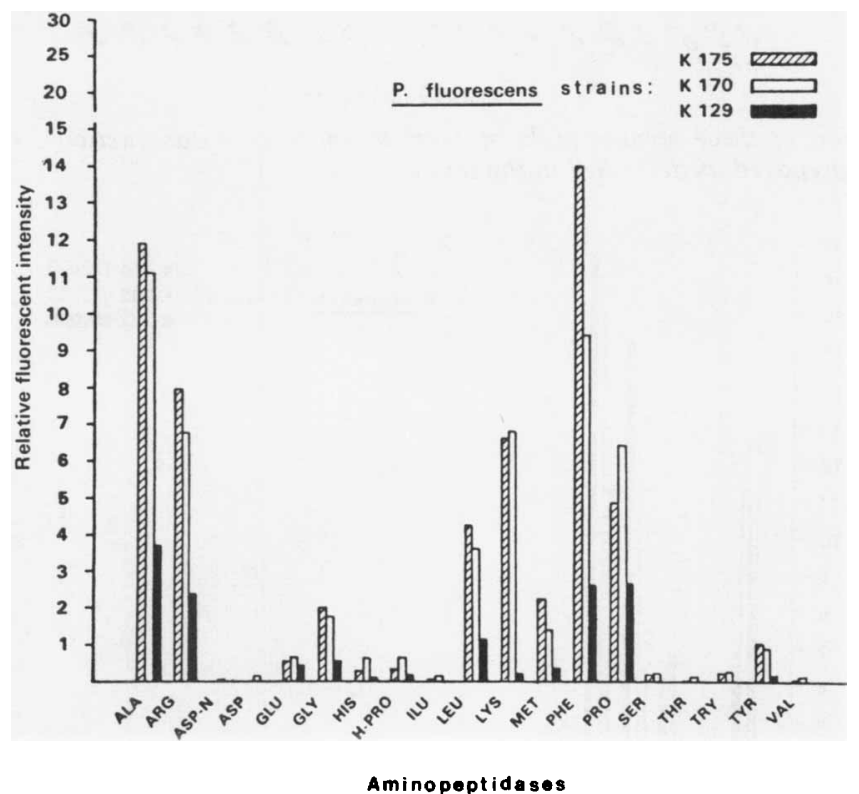

FIG. 3. Differentiation of three strains of $P$. fluorescens by aminopeptidase activity. Cells were grown in complex medium and prepared as described in the text.

its order of attack on the AA-NA substrates, making strain identification possible with these species.

$P$. stutzeri strains were characterized by their order of attack on the AA-NA substrates as follows: alanine, lysine, arginine, leucine, proline, methionine, glycine, phenylalanine, histidine, and tyrosine (Fig. 4). Their relatively low phen- 
ylalanine aminopeptidase activities were also characteristic.

As a group, the $P$. pickettii strains produced the most distinctive profiles (Fig. 5), with the following order of attack: glutamate, aspartate, alanine, asparagine, lysine, arginine, glycine, leu- cine, methionine, and serine. The high leucine, methionine, phenylalanine, and proline aminopeptidase activities of the strains of the three previously mentioned species were replaced by high glutamate, aspartate, and asparagine aminopeptidase activities (Fig. 2, 3, and 4). All three

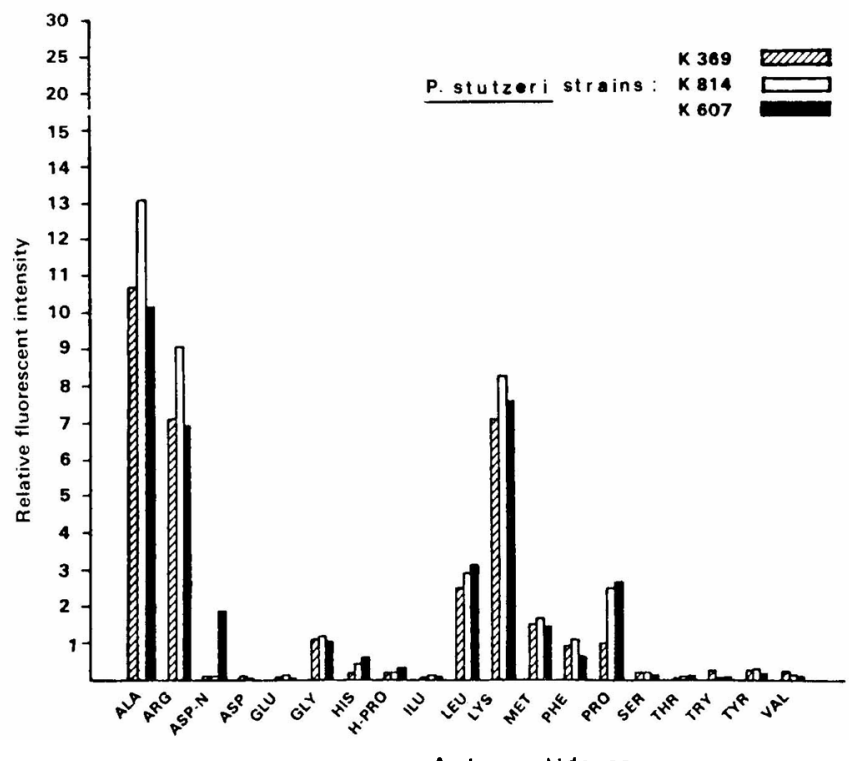

Aminopeptidasos

Fig. 4. Differentiation of three strains of P. stutzeri by aminopeptidase activity. Cells were grown in complex medium and prepared as described in the text.

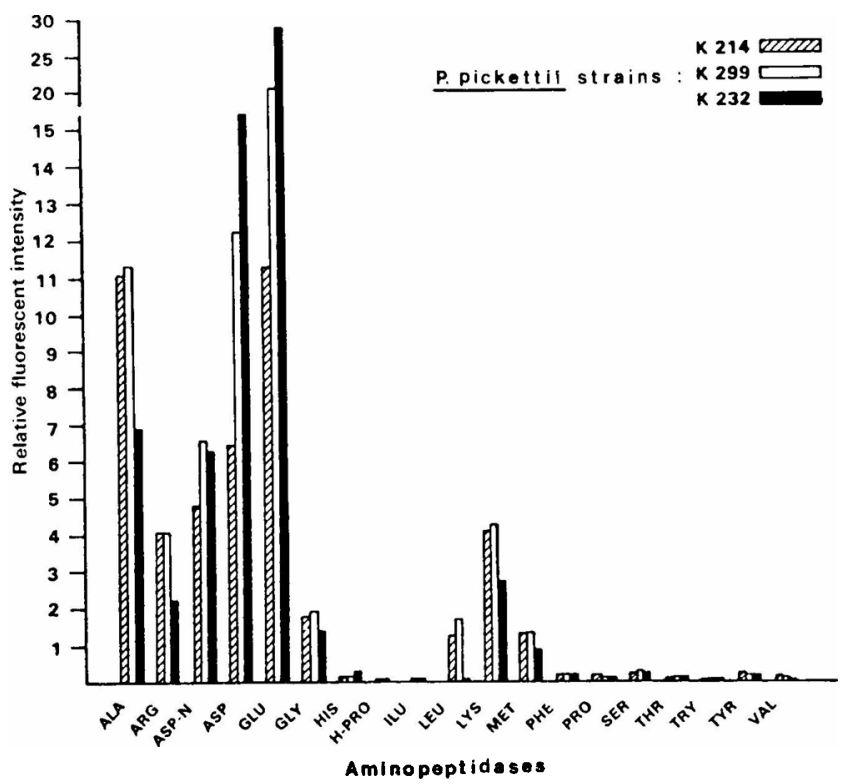

FIG. 5. Differentiation of three strains of P. pickettii by aminopeptidase activity. Cells were grown in complex medium and prepared as described in the text. 
$P$. pickettii strains consistently produced this unique pattern of substrate hydrolysis.

Aminopeptidase profiles in minimal medium. To determine the effect of different media on the aminopeptidase profiles, each microorganism was shifted down from the complex medium to the minimal medium. When assayed in the minimal medium, all strains showed two- to seven-fold amplification in most of the characteristic aminopeptidase activities (Fig. 6). This amplification added another parameter to the identification of the Pseudomonas strains in the present study. Examination of the minimal-medium profiles revealed that several of the strains had an increase in aminopeptidase activity of three-fold or greater (Fig. 7) than that observed for the previous medium (Fig. 1). P. fluorescens strains K129, K170, and K466 all had an increase in their phenylalanine aminopeptidase activity of more than 10-fold (data not shown for K129 and K170). P. stutzeri strains K369, K607, and $\mathrm{K} 814$ had increases in their proline aminopeptidase activities of five-, two-, and three-fold respectively (data not shown for K369 and K814). All four strains of $P$. putida produced only amplified profiles without any change in the observed pattern. The four aminopeptidases having the highest activity in nutrient broth more than doubled in activity in the minimal medium.
$P$. pickettii strain K232 responded differently by decreasing in its aspartate and glutamate aminopeptidase activity and increasing in its alanine, lysine, and methionine aminopeptidase activities.

In examining the pattern of attack exhibited by the 14 strains grown in minimal medium, it was found that the specificities of the aminopeptidases changed. The order in which the pseudomonads now preferentially hydrolyzed the AA-NA substrates was altered in each strain. $P$. putida K331 showed only three alterations in its pattern of substrate hydrolysis, whereas $P$. fluorescens strain $\mathrm{K} 466$ showed seven. This change in specificity resulted in the amplification of characteristic aminopeptidase activity and the emergence of different hydrolysates or in a decrease in the activity of other aminopeptidases. These observations suggest the need for using two growth media in conjunction with each other for the identification of the test organisms used in this investigation.

GLC analysis. The identifications of the Pseudomonas strains by GLC analysis were based on the profile of butyl ester derivatives of carboxylic acids produced as metabolic products. The identifications of these acids were achieved by comparing retention times of esterified acids in the sample to those of highly purified acid

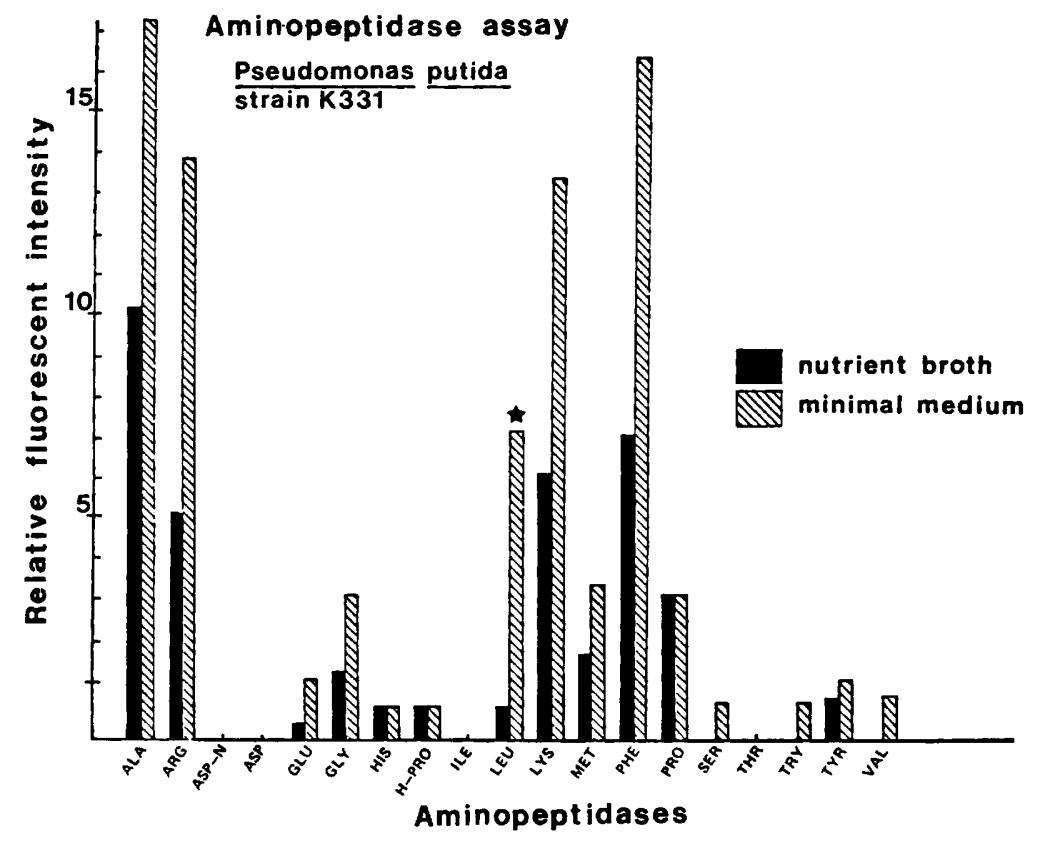

Fig. 6. Amplification of aminopeptidase profiles in the minimal medium. P. putida K331 aminopeptidase profiles compared after growth in nutrient broth and the minimal medium. A sevenfold increase in activity was seen in the hydrolysis rate for leucyl- $\beta$-naphthylamide when the microorganism was shifted down to the minimal medium. 


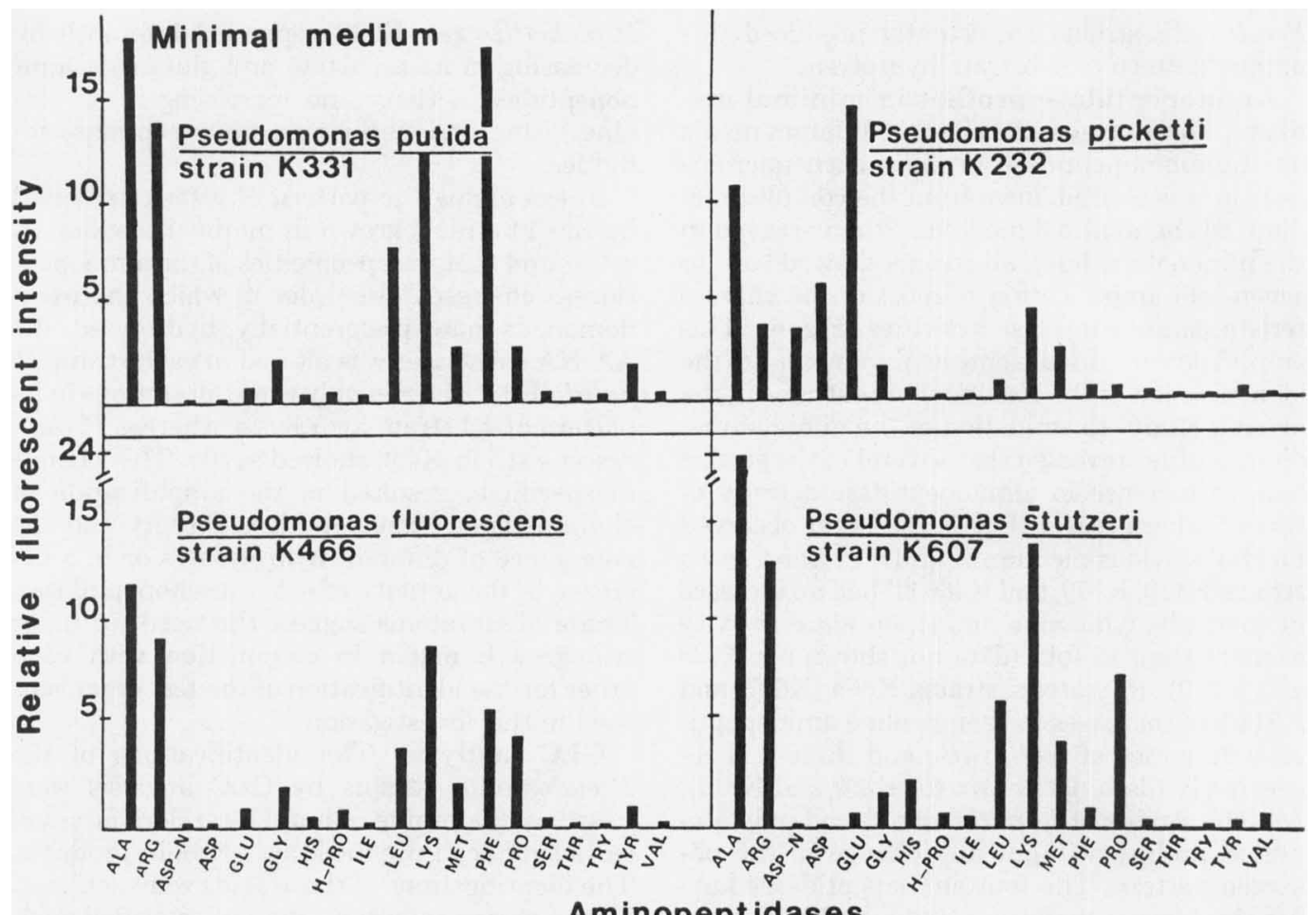

Aminopeptidases

FIG. 7. Differentiation of Pseudomonas strains by aminopeptidase activity. Cells were grown in the complex medium and then shifted down to the minimal medium. Note the increase in aminopeptidase activity after the. shift down.

standards. The four groups of strains thus profiled were easily distinguishable by GLC.

The strains were grown both in complex and minimal media and under aerated and nonaerated conditions to determine the conditions for most characteristic profiles. Trypticase soy broth (BBL Microbiology Systems, Cockeysville, $\mathrm{Md}$.) was also examined as a possible complex medium. Analysis of the uninoculated Trypticase soy broth control by GLC gave a chromatographic profile which was far too complex to be easily utilized as a control. Nutrient broth, however, fulfilled both requirements for a control medium of this type: it was an ideal growth medium for the test organisms used, and it had relatively few volatile components with retention times less than $20 \mathrm{~min}$. The four distinguishable compounds in the control, which eluted after $20 \mathrm{~min}$, were all utilized and disappeared in the cultures. Besides several minor peaks, the nutrient broth control had a rather large reagent peak which eluted from the column at $10 \mathrm{~min}$ (Fig. 8). This peak was due to one of the butylating steps as described previously (3).

$P$. putida K331 showed little acid production in nutrient broth under stationary or agitated conditions. Substrate utilization could be seen by comparing the uninoculated nutrient broth control with the cultured medium. The agitated and stationary cultures clearly produced different metabolic profiles of the same microorganism under different physiological conditions.

Acetate was chosen as a carbon source for the minimal medium because many pseudomonads have the ability to utilize this $\mathrm{C}_{2}$ compound as the sole source of carbon and energy. The chromatogram of the uninoculated minimal medium control showed only an acetate peak, a reagent peak, and a small contaminant peak appearing at 19 min (Fig. 9). The minimal medium control was excellent for making comparisons because of its nearly clear background. The metabolism of acetate was detected by several of the chromatograms, wherein the peak acetate area had been drastically reduced. The chromatograms of the agitated and stationary cultures in minimal medium showed several distinct differences in the metabolic patterns produced by $P$. putida K331. The stationary culture produced lactic and valeric acids, along with several unidentified acids which eluted later than $20 \mathrm{~min}$. Little reduction in the acetate peak indicated slow 


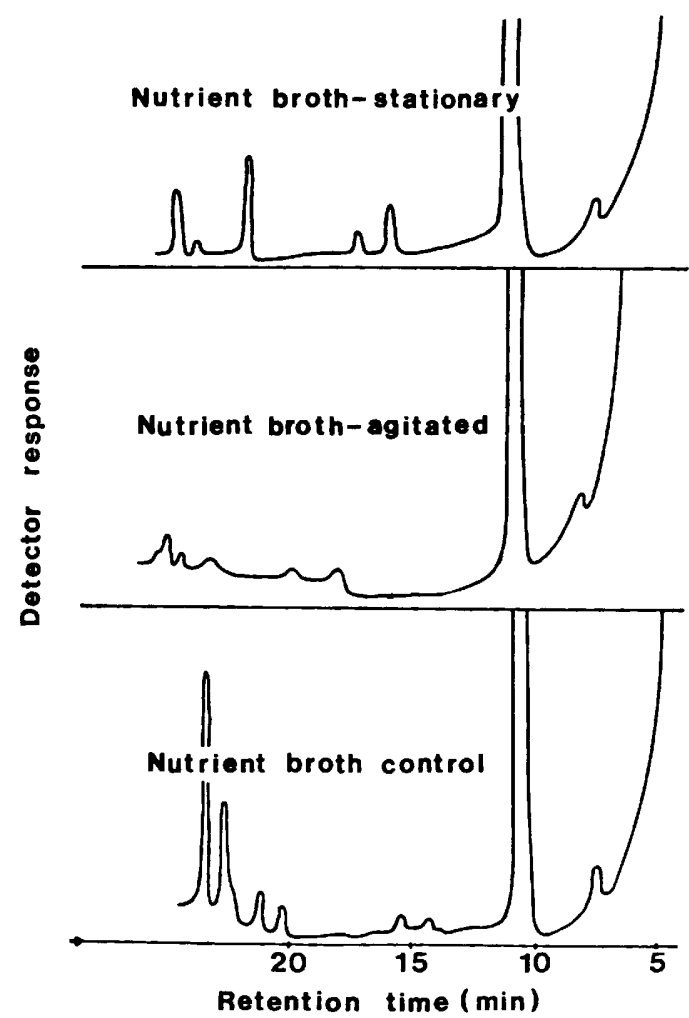

FIG. 8. GLC analysis of P. putida K331 grown in nutrient broth. Butyl ester derivatives were prepared from supernatants of aerated and nonaerated cultures.

metabolism by the microorganism under nonaerated conditions. The chromatogram of the agitated culture revealed the accelerated metabolism of acetate. The production of lactic, valeric, and heptanoic acids was also evident, with valeric acid production showing a significant increase.

Because of the distinct differences between the uninoculated control and the inoculated sample and the increased production of the short-chain carboxylic acids, the agitated minimal medium was chosen as the standard condition by which the Pseudomonas strains were identified (Fig. 10).

P. putida K331 was characterized by its ability to metabolize acetate at a high rate and to produce lactic, valeric, and heptanoic acids. Two minor unidentified peaks also appeared at the end of the chromatogram.

$P$. pickettii K232 gave the simplest chromatographic pattern of the strains of the four species. Only two acids, lactic and caproic, were produced by the $P$. pickettii strains. The slow me- tabolism of acetate was probably a contributing factor.

$P$. fluorescens K466 showed increased utilization of acetate, which correlated with the fact that strains of this species had the highest growth rate in the minimal medium. $P$. fluorescens $\mathrm{K} 466$ also produced the greatest number of acids, including butyric, lactic, caproic, and heptanoic acids and a significant amount of another acid, presently unidentified.

$P$. stutzeri K607 proved to be the most prolific producer of valeric acid. Along with valeric acid, this strain produced lactic acid and an unidentified acid which eluted from the column at 23 $\mathrm{min}$. This organism also had the ability to metabolize acetate at a high rate.

\section{DISCUSSION}

Both GLC analysis and aminopeptidase assay gave characteristic profiles of strains of four Pseudomonas species. Under strictly controlled conditions, the profiles of the test organisms

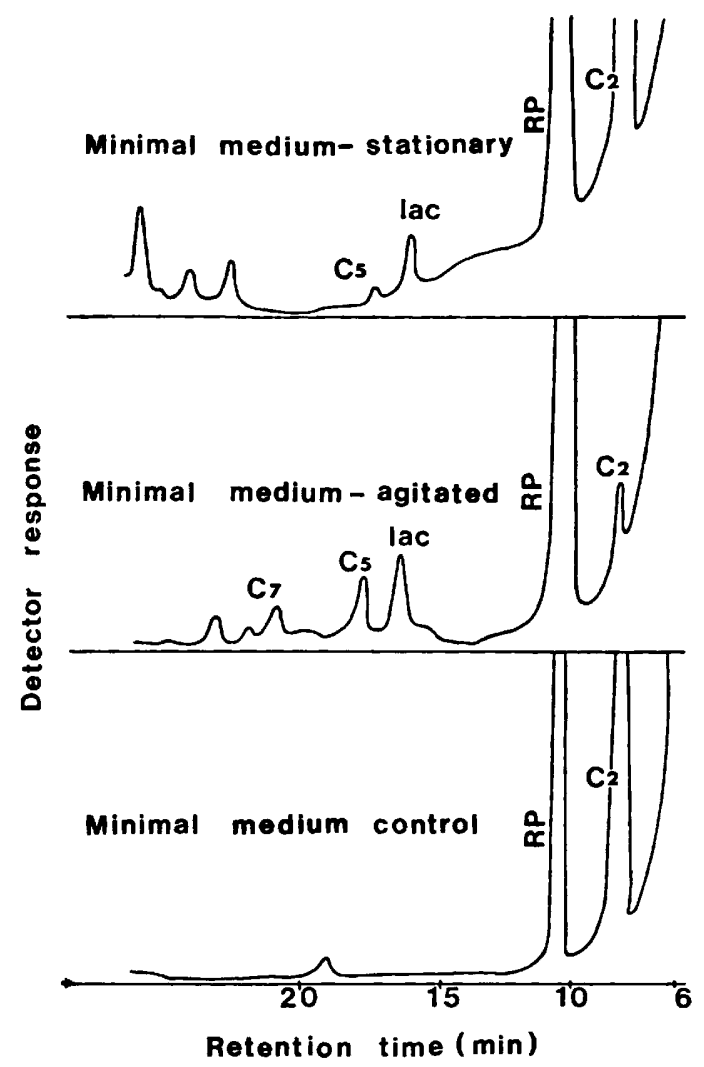

FIG. 9. GLC analysis of P. putida K331 grown in minimal medium. Butyl ester derivatives were prepared from supernatants of aerated and nonaerated cultures. 


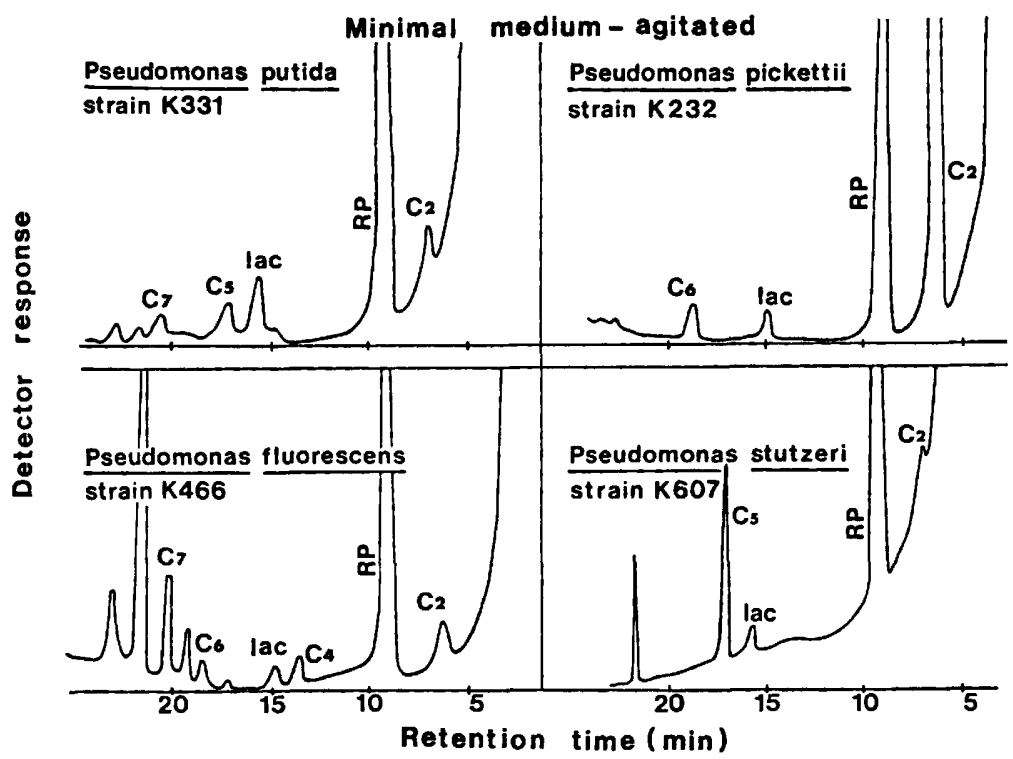

FIG. 10. Species differentiation of pseudomonads by GLC analysis. Each profile consists of metabolic end products of the microorganism and a reagent peak $(R P)$ which is an artifact of the butylation procedure.

proved reproducible through repeated experiments. Each procedure has been used in the past to separate and identify bacterial species not easily identified by conventional means $(5,9,15$, $21,27)$. Currently the characters used to identify nonfermentative rods as members of the genus Pseudomonas are the oxidase reaction, flagellation, nutritional requirements, composition of chromosomal deoxyribonucleic acid, and transformation $(17,22)$. Of these, the last two are not commonly determined in clinical laboratories. Because pseudomonads are capable of utilizing a vast array of organic compounds as nutrients, the number of tests conducted on nutritional requirements may reach as high as 100 or greater $(22,23)$. Cross-profiling pseudomonads with aminopeptidase assays and GLC analysis could aid in reducing the number of and the time needed for diagnostic tests.

Aminopeptidase assay. The profiles of the 14 Pseudomonas strains assayed in nutrient broth characteristically have high alanine and arginine aminopeptidase activities. The strains of $P$. putida, $P$. fluorescens, $P$. stutzeri, and $P$. pickettii were distinguished on the basis of their order of attack on the first 10 most actively hydrolyzed substrates. On the basis of their aminopeptidase profiles, the $P$. putida, $P$. fluorescens, and $P$. stutzeri strains were much more closely related to each other than they were to the $P$. pickettii strains (Fig. 2, 3, 4, and 5). The $P$. pickettii strains exhibited high glutamate, aspartate, and asparagine aminopeptidase activities not seen in the strains of the other three species. However, the results for strains of $P$. fluorescens (Fig. 3) were not very similar to those in Fig. 1, thus making it difficult to distinguish among these strains.

Comparison of the aminopeptidase profiles from the minimal medium with those from the complex medium revealed an amplification of characteristic aminopeptidase activities in the former. Amplification was often accompanied by changes in the pattern of preferential substrate hydrolysis. Westley et al. (27) determined that there were two major aminopeptidases in Bacillus mycoides, one of which was specific for leucine-napthylamide and the other of which was specific for phenylalanine, lysine, arginine, and probably methionine. The activity of each aminopeptidase was apparently repressed by the addition of leucine or any one of the latter three amino acids to the minimal medium.

One other possible explanation of the amplification which results from the shift to the minimal medium is the increased requirement for certain aminopeptidases during the turnover of preexisting cell components. After the shift to an environment which contains no amino acids, the microorganism must break down polypeptides and proteins by an increase in aminopeptidase biosynthesis.

The difference between profiles of complex and minimal medium cultures adds an addi- 
tional parameter to the identification of Pseudomonas strains. For example, the remarkable resemblance between strains of $P$. putida (Fig. 2) and strains of $P$. fluorescens (Fig. 3) makes it difficult to distinguish these two groups of strains grown in the complex medium. It is, therefore, essential to observe the characteristic amplification (Fig. 6) and the degree of amplification (Fig. 7) in the shift down experiment. The concurrent use of GLC analysis before and after the shift is also inevitable. Aminopeptidase profiles could also be used to prepare chemically defined media with near-perfect amino acid requirements for specific strains of bacteria.

GLC analysis. GLC analysis of biological samples has rapidly become a standard procedure in many clinical laboratories. Recent advances in derivative methods have allowed GLC researchers to chromatograph only the particular class of organic compounds to be examined (4).

High sensitivity is another advantage of the GLC analysis. Depending on the type of detector employed and the compound to be detected, minute quantities of components can be analyzed by GLC. Mitruka (13) used an electron capture detector to measure nanogram quantities of microbial metabolites. The flame ionization detector utilized by most GLC researchers is capable of detecting in the parts-per-million range.

Identification of unknown compounds has been accomplished by "splitting" the column effluent between the detector and a collector. To obtain pure samples, compounds are collected only during the detection of a single peak. Analytical methods such as infrared spectroscopy, mass spectrometry, nuclear magnetic resonance, and thin-layer and column chromatography are used to determine the chemical structure of the unknown compound. Systems have now been developed in which infrared or mass spectrometers are coupled to the gas chromatograph. Analysis and storage of data produced by such systems again is ideally handled by automatic data processing techniques.

Quantitative analysis can be carried out by the use of internal standards. Unlike external standards, which are injected separately and which may vary in relative retention times, internal standards are incorporated into the actual sample (20). An internal standard compensates for variations between runs in column temperatures, gas flow rates, detector sensitivity, sample preparation, and injection of different amounts of sample. Because a computerized digital integrator was not available to make the proper calculations required for an internal standard, a high degree of semi-quantitation was sought in the present study by maintaining certain controls. All cultures were grown in the same lot of medium, optical density readings were made to monitor the cell numbers, and a constant volume of sample was derivatized and injected onto the column.

The efficiency with which volatile components are recovered from spent medium is a major problem with GLC analysis. Previously, various extraction and distillation procedures have been used to remove volatile and nonvolatile acids from biological samples. Salanitro and Muirhead (20) noted that both procedures result in significant losses of volatile components, including formic, acetic, propionic, and iso-butyric acids. The ionization step used in this butylation procedure converts all acids immediately into sodium salts, which are recovered by freeze-drying of the alkaline medium.

Ionization increases recovery of highly volatile components by greatly reducing losses due to evaporation. Butylation itself enables one to chromatograph both volatile and nonvolatile carboxylic acids on the same column. In the past (13), volatile acids were chromatographed after a free acid extraction, whereas nonvolatile acids were chromatographed as methyl esters. Free acids tend to dimerize, adsorb onto the column, or catalyze reactions on the surface of metal columns (10). The butyl ester derivatization requires an 8-h preparation period, with a maximum number of 12 samples being prepared at one time. An additional $0.5 \mathrm{~h}$ is then required for each GLC analysis.

Analysis of spent medium has become the most popular method for profiling bacterial end products. External standards of acids found in these biological samples give easily reproduced profiles $(4,10,21)$. A standard medium such as nutrient broth can be purchased by any laboratory wanting to use the procedure. The composition of the acetate minimal medium was kept as simple as possible to enhance reproducibility.

$P$. fluorescens strains produced the greatest number of acids (six) in the minimal medium, whereas $P$. pickettii strains produced only two, lactic and caproic acids. None of the pseudomonads tested produced formic, propionic, or iso-butyric acid when grown in minimal medium. Growth rates for pseudomonads were higher in nutrient broth, but the minimal medium was chosen as the standard medium because the uninoculated control medium gave a negative background easily used in comparisons. $P$. putida strains produced a total of five acids in the minimal medium, three of which were lactic, valeric, and heptanoic acids. The $P$. stutzeri 
TABLE 1. Differentiation of pseudomonads by GLC analysis of butylester derivatives of acid end products ${ }^{a}$

\begin{tabular}{|c|c|c|c|c|c|c|c|c|c|c|c|c|}
\hline \multirow[b]{2}{*}{ Strain } & \multicolumn{12}{|c|}{ Carboxylic acid } \\
\hline & $\mathbf{C}_{1}$ & $\overline{\mathrm{C}_{2}}$ & $\overline{\mathrm{C}_{3}}$ & $\mathrm{i}-\mathrm{C}_{4}$ & $\mathrm{C}_{4}$ & Lactic & $\overline{C_{5}}$ & $\mathrm{U}_{1}^{b}$ & $\overrightarrow{\mathrm{C}_{6}}$ & $\overline{\mathrm{U}_{2}^{b}}$ & $\mathrm{C}_{7}$ & $\mathrm{U}_{3}^{b}$ \\
\hline \multicolumn{13}{|c|}{ P. fluorescens } \\
\hline K170 & $-^{c}$ & + & - & - & + & + & - & - & + & + & + & + \\
\hline K175 & - & + & - & - & + & + & - & - & + & - & + & + \\
\hline K466 & - & + & - & - & + & + & - & - & + & + & + & + \\
\hline K129 & - & + & - & - & + & + & - & - & - & + & + & - \\
\hline \multicolumn{13}{|l|}{ P. putida } \\
\hline K89 & - & + & - & - & + & + & + & - & - & + & + & + \\
\hline K355 & - & + & - & - & + & + & + & - & - & + & + & - \\
\hline K360 & - & + & - & - & + & + & + & - & - & + & + & + \\
\hline K331 & - & + & - & - & + & + & + & - & - & + & + & - \\
\hline \multicolumn{13}{|l|}{ P. stutzeri } \\
\hline K369 & - & + & - & - & - & + & + & - & - & + & + & - \\
\hline K607 & - & + & - & - & - & + & + & - & - & - & - & + \\
\hline K814 & - & + & - & - & - & + & + & - & + & - & + & + \\
\hline \multicolumn{13}{|l|}{ P. pickettii } \\
\hline K214 & - & + & - & - & - & + & - & - & + & - & - & - \\
\hline K279 & - & + & - & - & - & + & - & - & + & - & - & - \\
\hline K232 & - & + & - & - & - & + & - & - & + & - & - & - \\
\hline
\end{tabular}

${ }^{a}$ Cells were grown in agitated minimal medium.

${ }^{b}$ Unidentified peaks.

${ }^{c}+$, Carboxylic acids produced as metabolic products; - , carboxylic acids not produced as metabolic products.

strains produced the greatest amounts of valeric acid in the minimal medium (Table 1).

Analysis of microbial metabolites by GLC can also be utilized to study in a single bacterial strain the changes in metabolism brought about by alterations in the environment. Profiles of $P$. putida K331 (Fig. 8) showed a significant decrease in the concentration of the carbon source (acetate) in the agitated cultures. No similar decrease was seen in the stationary culture. Profiles from minimal medium cultures did show an increase in the quantity of short-chain acids. Agitated minimal medium cultures produced large amounts of lactic and valeric acids. Production of valeric acid was significantly reduced in the stationary culture.

In exploring possibilities for the use of GLC analysis in areas other than bacterial identification, Mitruka (13) devised techniques in which bacterial infections, viral infections, disease states, and metabolic deficiency diseases could be identified.

In cross-profiling bacterial strains, one reached the ultimate economic situation of utilizing both the microorganism and its extracellular products to obtain two metabolic profiles, both of which are characteristic of that microorganism. One disadvantage of this procedure is that it is limited to pure cultures; mixed-culture analysis is not within the scope of this work. Automated systems such as GLC analysis and aminopeptidase assays will someday provide the technology to develop a system capable of ac- curate and rapid identification of microorganisms.

\section{ACKNOWLEDGMENTS}

We thank J. L. Valentine and R. Holland for their technical assistance.

J.H.G. was supported by a faculty research grant from the University of Missouri-Kansas City.

\section{REPRINT REQUESTS}

Address reprint requests to: Dr. Edward J. Hsu, Department of Biology, University of Missouri, 5100 Rockhill Rd., Kansas City, MO 64110.

\section{LITERATURE CITED}

1. Choules, G. L., and W. R. Gray. 1971. Peptidase activity in the membranes of Mycoplasma laidlawii. Biochem. Biophys. Res. Comm. 45:849-855.

2. Chakrabarty, A. M. 1976. Plasmids in Pseudomonas. Annu. Rev. Genet. 10:7-30.

3. Doelle, H. W. 1969b. Gas chromatographic separation and determination of microquantities of $\mathrm{C}_{1}-\mathrm{C}_{7}$ branched and straight chain saturated fatty acids. J. Chromatogr. 39:398-406.

4. Drucker, D. B. 1976. Gas-liquid chromatographic chemotaxonomy, p. 51-125. In J. R. Norris and D. W. Ribbons (ed.), Methods in microbiology, vol. 9. Academic Press, Inc. New York.

5. Fugate, K. J., L. B. Hanse, and O. White. 1971. Analysis of Clostridium botulinum toxigenic types A, B, and E for fatty acid and carbohydrate content. Appl. Microbiol. 21:470-475.

6. Garner, C. W., and F. J. Behen. 1975. Human liver alanine aminopeptidase. Inhibition by amino acids. Biochemistry 14:3208-3212.

7. Graevenitz, A. V., and J. Weinstein. 1971. Pathogenic significance of Pseudomonas fluorescens and Pseudomonas putida. Yale J. Biol. Med. 44:265-273. 
8. Kageyama, M., Y. Sano, and T. Shinomiya. 1979. Suppressor mutation in Pseudomonas aeruginosa. J. Bacteriol. 138:748-755.

9. Kimble, C. E., M. L. McCollough, V. A. Paterno, and A. W. Anderson. 1969. Comparisons of the fatty acids of proteolytic type $B$ and nonproteolytic types $E$ and $F$ of Clostridium botulinum. Appl. Microbiol. 18:883-888.

10. Lambert, M. A., and C. W. Moss. 1972. Gas-liquid chromatography of short-chain fatty acids on Dexsil 300-GC. J. Chromatogr. 74:335-338.

11. Matheson, A. T., A. J. Dick, and F. Rollin. 1970. A ribosomal-bound aminopeptidase in Escherichia coli $\mathrm{B}$ : substrate specificity. Can. J. Biochem. 48:1292-1296.

12. Mayhew, J. W., and S. L. Gorbach. 1975. Rapid gas chromatographic technique for presumptive detection of Clostridium botulinum in contaminated foods. Appl. Microbiol. 29:297-299.

13. Mitruka, B. M. 1974. Gas chromatography applications in microbiology and medicine. John Wiley \& Sons, Inc., New York.

14. Moss, C. W., and S. B. Samuels. 1974. Short-chain acids of Pseudomonas species encountered in clinical specimens. Appl. Microbiol. 27:570-574.

15. Peterson, E. H., and E. J. Hsu. 1978. Rapid detection of selected gram-negative bacteria by aminopeptidase profiles. J. Food Sci. 43:1853-1856.

16. Pickett, M. J., and M. M. Pederson. 1968. Characterization of bacteria by their degradation of amino acids. Appl. Microbiol. 16:1591-1595.

17. Pickett, M. J., and M. M. Pederson. 1970a. Characterization of saccharolytic non-fermentative bacteria associated with man. Can. J. Microbiol. 16:351-362.

18. Ralston, E., N. J. Palleroni, and M. Doudoroff. 1973. Pseudomonas pickettii, a new species of clinical origin related to Pseudomonas solanacearum. Int. J. Syst.
Bacteriol. 23:15-19.

19. Rose, B., J. M. Becker, and F. Naider. 1979. Peptidase activities in Saccharomyces cerevisiae. J. Bacteriol. 139:220-224.

20. Salanitro, J. P., and P. A. Muirhead. 1975. Quantitative method for the gas chromatographic analysis of shortchain monocarboxylic and dicarboxylic acids in fermentation media. Appl. Microbiol. 29:374-381.

21. Samuels, S. B., C. W. Moss, and R. E. Weaver. 1973. The fatty acids of Pseudomonas multivorans (Pseudomonas cepacia), and Pseudomonas kingii. J. Gen. Microbiol. 74:275-279.

22. Sands, D. C., M. N. Schroth, and D. C. Hildebrand. 1970. Taxonomy of phytopathogenic pseudomonads. $J$. Bacteriol. 101:9-23.

23. Stanier, R. Y., N. J. Palleroni, and M. Doudoroff. 1966. The aerobic pseudomonads: a taxonomic study. J. Gen. Microbiol. 43:159-271.

24. Wade, T. J., and R. J. Mandle. 1974.' New gas chromatographic characterization procedure: preliminary studies on some Pseudomonas species. Appl. Microbiol. 27:303-311.

25. Wagner, F. W., S. H. Wilkes, and J. M. Prescott. 1972. Specificity of Aeromonas aminopeptidase toward amino acids and dipeptides. J. Biol. Chem. 247:12081210.

26. Watson, R. R. 1976. Substrate specificities of aminopeptidases: a specific method for microbial differentiation, p. 1-13. In J. R. Norris and D. W. Ribbons (ed.), Methods in microbiology, vol. 9. Academic Press, Inc., New York.

27. Westley, J. W., P. J. Anderson, V. A. Close, B. Halpern, and E. M. Lederberg. 1967. Aminopeptidase profiles of various bacteria. Appl. Microbiol. 15:822825. 